\title{
Adenosine deaminase deficiency increases thymic apoptosis and causes defective $T$ cell receptor signaling
}

\author{
Sergey G. Apasov, ${ }^{1}$ Michael R. Blackburn, ${ }^{2}$ Rodney E. Kellems, ${ }^{2}$ Patrick T. Smith, ${ }^{1}$ \\ and Michail V. Sitkovsky ${ }^{1}$ \\ ${ }^{1}$ Laboratory of Immunology, National Institute of Allergy and Infectious Diseases, NIH, Bethesda, Maryland, USA \\ ${ }^{2}$ University of Texas-Houston Medical School, Department of Biochemistry and Molecular Biology, Houston, Texas, USA \\ Address correspondence to: Michail V. Sitkovsky, Laboratory of Immunology, National Institutes of Health, \\ 10/11N311, 10 Center Drive, Bethesda, Maryland 20892-1892, USA. \\ Phone: (301) 496-5495; Fax: (301) 480-7374; E-mail: mvsitkov@ helix.nih.gov.
}

Received for publication May 17, 2000, and accepted in revised form May 14, 2001.

\begin{abstract}
Adenosine deaminase (ADA) deficiency in humans results in a severe combined immunodeficiency (SCID). This immunodeficiency is associated with severe disturbances in purine metabolism that are thought to mediate lymphotoxicity. The recent generation of ADA-deficient $\left(A D A^{-/}\right)$mice has enabled the in vivo examination of mechanisms that may underlie the SCID resulting from ADA deficiency. We demonstrate severe depletion of $\mathrm{T}$ and $\mathrm{B}$ lymphocytes and defects in $\mathrm{T}$ and $\mathrm{B}$ cell development in $A D A^{-/-}$mice. T cell apoptosis was abundant in thymi of $A D A^{-/-}$mice, but no increase in apoptosis was detected in the spleen and lymph nodes of these animals, suggesting that the defect is specific to developing thymocytes. Studies of mature T cells recovered from spleens of $A D A^{-/-}$mice revealed that ADA deficiency is accompanied by TCR activation defects of T cells in vivo. Furthermore, ex vivo experiments on $A D A^{-/-} \mathrm{T}$ cells demonstrated that elevated adenosine is responsible for this abnormal TCR signaling. These findings suggest that the metabolic disturbances seen in $A D A^{-/-}$ mice affect various signaling pathways that regulate thymocyte survival and function. Experiments with thymocytes ex vivo confirmed that ADA deficiency reduces tyrosine phosphorylation of TCRassociated signaling molecules and blocks TCR-triggered calcium increases.
\end{abstract}

J. Clin. Invest. 108:131-141 (2001). DOI:10.1172/JCI200110360.

\section{Introduction}

Inherited immunodeficiencies were recognized as distinct human disease entities several decades ago. In subsequent years, the analysis of naturally occurring immunodeficiencies yielded considerable insight regarding the composition and function of the immune system. In 1972, adenosine deaminase (ADA) deficiency became the first of the immunodeficiency diseases for which a specific molecular defect was identified (1). The importance of ADA for immune function was revealed unexpectedly when it was discovered that the enzyme was absent in several immunodeficient patients being considered for bone marrow transplantation therapy. Within a few years of this discovery, it was determined that a deficiency in another purine catabolic enzyme, purine nucleoside phosphorylase (PNP), also results in a serious immunodeficiency disease in humans, although in this case the lymphotoxic impact is largely restricted to $T$ cells (2). Together, these findings underscore the importance of these two metabolically linked purine catabolic enzymes to proper immune development and/or function. Because of its higher prevalence and more profound immunological impact, more attention has been focused on ADA deficiency.

ADA deficiency is one of the most severe of the immunodeficiencies in humans and is associated with a severe depletion of all three major categories of lym- phocytes, T cells, B cells, and NK cells (3). ADA deficiency is the second-most prevalent form of severe combined immunodeficiency disease (SCID), accounting for approximately $20 \%$ of the group $(3,4)$. Without intervention, affected individuals die from overwhelming opportunistic infections within the first few months of life. Available evidence suggests that the metabolic basis for ADA-deficient immunodeficiency is related to the impact of its substrates, adenosine and $2^{\prime}$-deoxyadenosine (reviewed in ref. 4). However, the exact molecular mechanisms of lymphotoxicity remain obscure, and the relative contribution of intracellular lymphotoxic versus signaling properties of extracellular adenosine on ADA SCID have not been sufficiently explored. Adenosine may function as an extracellular signal transducer that mediates a variety of physiological effects by binding to $G$ protein-coupled receptors present on the surface of target cells (5). In this regard, the accumulation of adenosine and the activation of adenosine receptors known to be present on T cells (6) may lead to impaired $\mathrm{T}$ cell development or function $(7,8)$. Alternatively, $2^{\prime}$-deoxyadenosine may act as a cytotoxic metabolite that can mediate its effects directly at the nucleoside level or after conversion to dATP. Potential downstream consequences of elevated 2'deoxyadenosine and/or dATP include: (a) interference with cellular transmethylation reactions (9), (b) inter- 
ruption of deoxynucleotide synthesis $(10,11)$, and (c) activation of apoptosis (12). Thus, ADA deficiency may provoke a variety of consequences either through metabolic disturbances caused by elevated 2 '-deoxyadenosine or cell signaling disturbances caused by elevated adenosine. Efforts to identify the metabolic and molecular basis for the immune dysfunction associated with ADA deficiency are motivated in part by the desire to identify pharmacological targets that could be used to control the immune system. This could be of considerable benefit in treating conditions where lymphocytes proliferate or function abnormally, such as lymphocytic leukemias and autoimmune disease. To facilitate the analysis of the SCID phenotype associated with ADA deficiency, we have recently created a mouse model of ADA-deficient SCID (13).

A two-stage genetic engineering strategy was used to produce ADA-deficient mice that retain many of the features associated with ADA deficiency in humans (13, 14). These mice develop a combined immunodeficiency that is associated with profound disturbances in purine metabolism (13). The ability to analyze tissues from ADA-deficient mice, an experimental approach not possible with ADA-deficient humans, enabled us to make novel immunological and metabolic observations. Analysis of tissue-associated metabolic disturbances in these mice revealed that absolute increases in ADA substrates are greatest in the thymus and spleen (15), suggesting that metabolic disturbances in these major immune organs play a major role in the ensuing immunodeficiency (13). Here we report a detailed analysis of the impact of ADA deficiency on intrathymic $\mathrm{T}$ cell development and on $\mathrm{T}$ cell receptor-mediated (TCR-mediated) signaling in the periphery. Our results show that ADA deficiency is associated with enhanced thymocyte apoptosis and impaired TCR signaling.

\section{Methods}

Animals. ADA-deficient mice $\left(A D A^{-/}\right)$were developed using a two-stage genetic engineering strategy (13). The development of this mouse model was based on previous studies that suggested that ADA expression in trophoblast cells of the placenta is critical for fetal development in the mouse (14). Thus, to generate completely ADA-deficient postnatal mice, an ADA minigene that targeted expression specifically to the trophoblast lineage, was introduced onto the ADAdeficient background (13). This was accomplished by intercrossing mice carrying the trophoblast-specific ADA minigene with mice heterozygous for the null ada allele. Subsequent intercrosses yielded litters that contained mice harboring the ADA minigene that were also homozygous for the null ada allele. Given that the regulatory elements used targeted ADA expression only to trophoblasts (14), once born, and with the loss of the placenta, ADA enzymatic activity was not observed in any of the tissues examined in $A D A^{-/-}$mice (13).

Mice were bred and maintained in the NIH pathogenfree animal facilities. $A D A^{-/-}$mice were first identified using ADA zymogram analysis of blood samples, and then genotypes were conclusively determined by Southern blot analysis of tail DNA (Figure 1a). Measurements of ADA activity in blood samples from littermates born to heterozygous $\left(A D A^{+-}\right)$intercrosses were performed by zymogram analysis (13) using a modified protocol of Knudsen et al. (16). Zymogram analysis was conducted with G493 agarose gels in a temperature-controlled electrophoresis chamber (Innovative Chemistry, Marshfield, Massachusetts, USA). Heat inactivation and ADA inhibitor treatment (coformycin; Calbiochem-Novabiochem International, San Diego, California, USA) prevented ADA band visualization in control samples, thereby verifying the ADA enzymatic activity assays in zymogram gels. ADA wildtype $\left(A D A^{+/+}\right)$and heterozygous $\left(A D A^{+/-}\right)$littermates were used as controls for most studies, as no differences between heterozygous and wild-type mice were found in preliminary experiments (data not shown).

Cells and medium. Thymocytes or spleen cells were isolated from 3-week-old mice and either directly labeled with mAb's or incubated in RPMI-1640 (Biofluids Inc., Rockville, Maryland, USA) supplemented with $5 \%$ dialyzed FCS (heat inactivated) and $100 \mathrm{U} / \mathrm{ml}$ penicillin, $100 \mu \mathrm{g} / \mathrm{ml}$ streptomycin, $1 \mathrm{mM}$ sodium pyruvate, 1 mM HEPES, nonessential amino acids, and $5 \times 10^{-5} \mathrm{M}$ $2 \beta$-mercaptoethanol. Where indicated, cells were incubated in ADA-free serum-free media (Life Technologies Inc., Gaithersburg, Maryland, USA).

Reagents. R-phycoerythrin-conjugated (R-PE-conjugated) rat anti-mouse anti-CD25 $\mathrm{mAb}$, anti-mouse CD69, and FITC-conjugated rat anti-mouse CD8a $\mathrm{mAb}$ 's, as well as Cy-Chrome-conjugated CD4, were purchased from PharMingen (San Diego, California, USA). Rat anti-mouse CD 4 mAb's conjugated with RED-613 fluorochrome were purchased from Life Technologies Inc. Adenosine was prepared freshly as $20 \mathrm{mM}$ stock solution. Adenosine and the ADA inhibitor EHNA were purchased from Sigma-Aldrich (St. Louis, Missouri, USA). 2'-Deoxyadenosine was purchased from Sigma Chemical Co. (St. Louis, Missouri, USA). Coformycin was purchased from Calbiochem-Novabiochem International. Annexin V-FITC was purchased from BioWhittaker Inc. (Walkersville, Maryland, USA).

Flow cytometry. Single-cell suspensions of murine thymocytes were generated by standard procedures, and cells were either directly analyzed or cultured in 96-well plates $\left(0.5 \times 10^{6}-1 \times 10^{6}\right.$ cells per well $)$ as described elsewhere (17). After incubation for 16-18 hours or as indicated, cells were harvested and analyzed by flow cytometry. Flow cytometric quantitation of live, apoptotic, and dead cells was done according to a modified flow cytometry procedure (18) as described previously (17). The effects of adenosine on thymocytes were studied after incubating thymocytes ex vivo in short-term culture. Determining the status of cells (live, dead, or apoptotic) was based on gating of cells by their size (side scatter and forward scatter), 


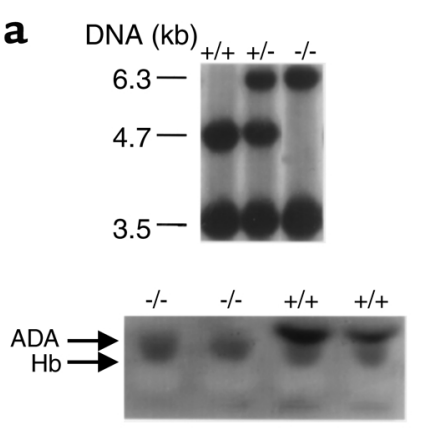

b

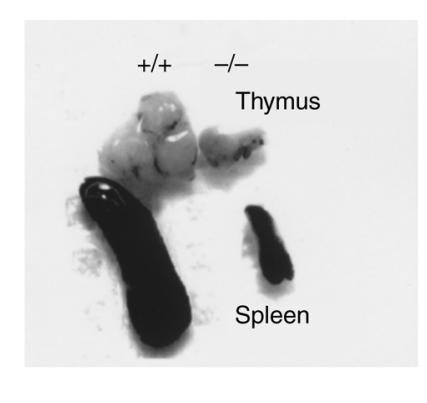

c

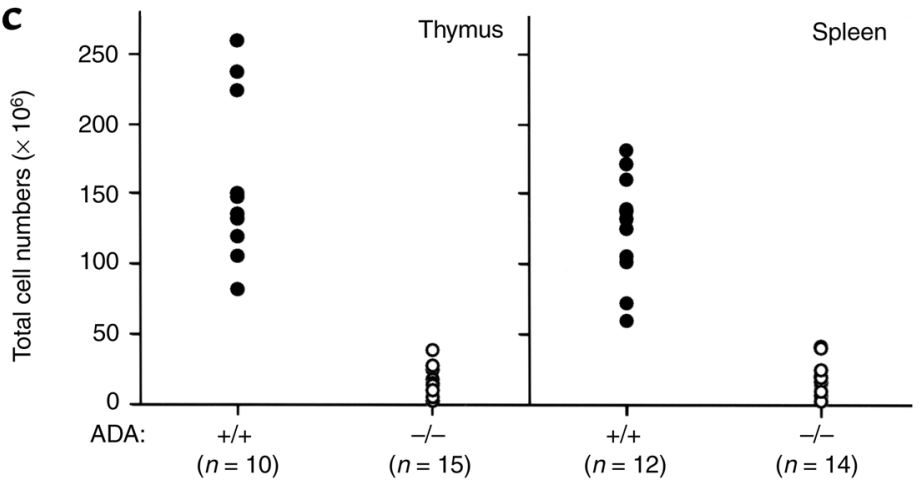

\section{Figure 1}

Demonstration of extensive cell death in thymus but not in peripheral lymphoid organs of $A D A^{-/-}$mice. (a) Genetic and biochemical evidence of ADA deficiency in screening for $A D A^{+/+}, A D A^{+/-}$, and $A D A^{-/-}$ mice. Littermates of ADA heterozygous mice were first analyzed by zymogram assay to identify $A D A^{-/-}$mice, and then tail DNA samples were analyzed by Southern blot as described in ref. 11. Arrowheads on the zymogram indicate position of ADA and hemoglobin (internal control). (b) Comparison of spleens and thymi from $A D A^{+/+}$and $A D A^{-/-}$littermates. $\times 3$. (c) Decreased cellularity of lymphoid organs of $A D A^{-/-}$ mice. $n$, number of animals analyzed. plasma membrane integrity (PI staining), and redistribution of plasma membrane phosphatidylserine (Annexin V-binding). The Annexin V binding assay was done as described previously (19). Briefly, $0.6 \times 10^{6}-1 \times 10^{6}$ cells were resuspended in $100 \mu \mathrm{l}$ of buffer containing $10 \mathrm{mM}$ HEPES ( $\mathrm{pH}$ 7.3), $150 \mathrm{mM}$ $\mathrm{NaCl}, 5 \mathrm{mM} \mathrm{KCl}, 1 \mathrm{mM} \mathrm{MgCl}$, and $1.8 \mathrm{mM} \mathrm{CaCl}_{2}$ and incubated with $0.3 \mu \mathrm{g} / \mathrm{ml}$ of FITC-conjugated Annexin $V$ and $5 \mu \mathrm{g} / \mathrm{ml}$ propidium iodide for $15 \mathrm{~min}$ utes. After incubation, samples were diluted four times with buffer containing $1.8 \mathrm{mM} \mathrm{CaCl}_{2}$ and were analyzed by FACScan (Becton Dickinson Immunocytometry Systems, San Jose, California, USA).

Statistical analysis of triplicate sample measurements was performed using the StatView statistic program (Abacus-Concepts Inc., Berkeley, California, USA). Standard deviations of triplicate measurements within the same experiment were lower than $1 \%$. Flow cytometry data acquisition and analysis were done on FACScan using FACScan research software and CellQuest programs (both, Becton Dickinson Immunocytometry Systems).

$\mathrm{Ca}^{2+}$ measurements. For measurements of $\mathrm{Ca}^{2+}$ flux, freshly isolated thymocytes were preloaded with indo1 (final $3 \mu \mathrm{M}$ ) in $\mathrm{Ca}^{2+}$ buffer (1\% FCS, $10 \mathrm{mM}$ HEPES, HBSS) for 30 minutes at $37^{\circ} \mathrm{C}$ and were washed and analyzed on a FACSVantage flow cytometer (Becton Dickinson Immunocytometry Systems) equipped with an argon laser tuned to $488 \mathrm{~nm}$ and a krypton laser tuned to $360 \mathrm{~nm}$.

Indo-1 fluorescence was analyzed at 390/20 and $530 / 20 \mathrm{~nm}$ for bound and free probe as was described elsewhere $(20,21)$. The percentage of cells that responded by an increase in intracellular $\mathrm{Ca}^{2+}$ after stimulation with Concanavalin A $(2.5 \mu \mathrm{g} / \mathrm{ml})$ (Vector Laboratories, Burlingame, California, USA) was determined using CellQuest software program.

In situ analysis of apoptosis in thymocytes. Frozen tissue preparations and apoptosis analysis in the spleen, thymus, and lymph nodes of $A D A^{+/+}$and $A D A^{-/-}$mice were performed according to procedures used by Molecular Histology Inc. (Gaithersburg, Maryland, USA). The detection of apoptotic cells by in situ staining of singlestrand breaks in nuclear DNA was preformed according to protocols described previously $(22,23)$.

Immunoblotting with anti-phosphotyrosine $m A b$ 's. After isolation, cells were incubated for 10 minutes at $37^{\circ} \mathrm{C}$, washed once with PBS, and placed in lysis buffer containing 1\% NP 40, $10 \mathrm{mM}$ Tris- $\mathrm{HCl}$ ( $\mathrm{pH} 7.2$ ), $140 \mathrm{mM}$ $\mathrm{NaCl}, 2 \mathrm{mM}$ EDTA, $5 \mathrm{mM}$ iodoacetamide, $1 \mathrm{mM}$ $\mathrm{Na}_{3} \mathrm{VO}_{4}$ (Sigma Chemical Co.), and complete protease inhibitor cocktail (Boehringer Mannheim GmbH (Mannheim, Germany) for 25 minutes on ice. After removal of nuclear debris by centrifugation, the resultant supernatants were subjected to immunoprecipitation and analyzed by immunoblotting. ZAP-70 immunoprecipitation SDS-PAGE and immunoblotting were performed as described previously (24). The following antibodies were used in these experiments: rabbit antiserum to ZAP-70 (31); 4G10, a mouse mAb to phosphotyrosine (Upstate Biotechnology Inc., Lake Placid, New York, USA) and peroxidase-linked goat antibodies to mouse and rabbit Ig (Bio-Rad Laboratories Inc., Richmond, California, USA). 


\section{Results}

Extensive apoptosis occurs in the thymus but not in lymph nodes or spleens of ADA-deficient mice. ADA deficiency in humans and mice results in lymphopenia $(3,13)$. Previously, the status of the immune system of $A D A^{-/-}$ mice was analyzed at 2 weeks of age, and, although a lymphopenia was seen, it was not severe (13). We therefore analyzed the immune status of $A D A^{-/-}$mice at 3 weeks of age to determine whether the immune status was more severe at these later stages. Figure 1 demonstrates that there was a pronounced decrease in the size of immune organs in $A D A^{-/-}$mice (Figure 1a). Furthermore, analysis of cells isolated from $A D A^{-/-}$thymi, spleens, and lymph nodes demonstrated a large reduction in the absolute numbers of cells found in $A D A^{-/-}$ immune organs (Figures 1, b and c). These data demonstrated that a severe lymphopenia was present in $A D A^{-/-}$mice at 3 weeks of age.

The metabolic consequences associated with ADA deficiency have been demonstrated to induce apopto- sis in lymphocytes in vitro $(25,26)$ suggesting that apoptosis may play an important role in the lymphopenia associated with ADA deficiency. To determine whether apoptosis was increased in lymphoid tissues in association with ADA deficiency in vivo, we analyzed lymphocytes in immune organs of $A D A^{-/-}$ mice for the presence of live, apoptotic, and dead cells (Figure 2). Analysis of side scatter versus forward scatter in thymi from $A D A^{-/-}$and $A D A^{+/+}$littermates revealed an increase in the number of dead cells among immature $\mathrm{T}$ cells isolated from $A D A^{-/-}$thymi (Figure $2 \mathrm{a}$, top), whereas the proportions of dead cells among mature $\mathrm{T}$ cells found in the peripheral lymphoid organs (spleen and lymph nodes) were similar between $A D A^{+/+}$and $A D A^{-/-}$mice (data not shown). The double staining of cells with PI and Annexin $\mathrm{V}$ reveals an increase in apoptosis in the thymus of $A D A^{-/-}$mice (Figure 2a, bottom), but not in peripheral lymph organs (data not shown). These data suggest that there was an increase in apoptosis in thymi of $A D A^{-/-}$mice, $\mathbf{a}$
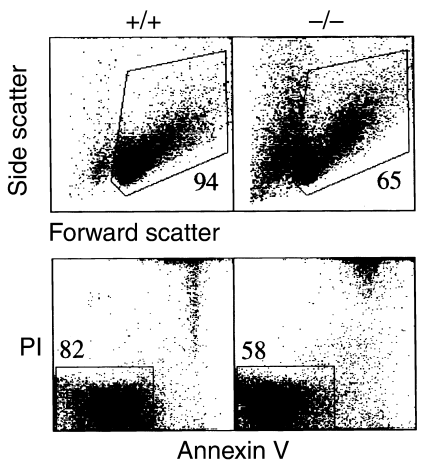

C

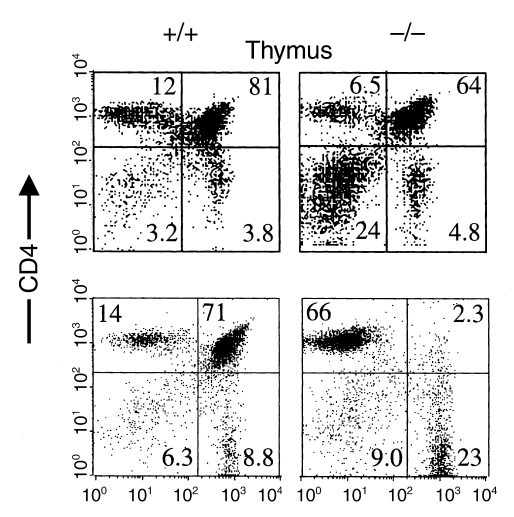

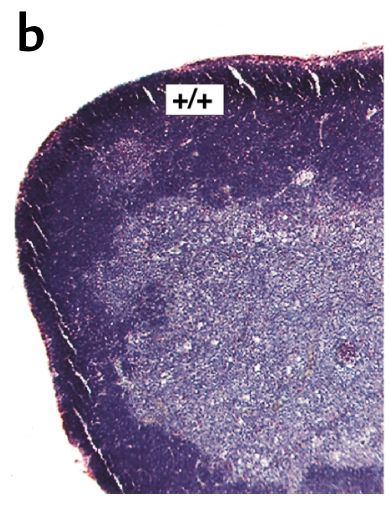

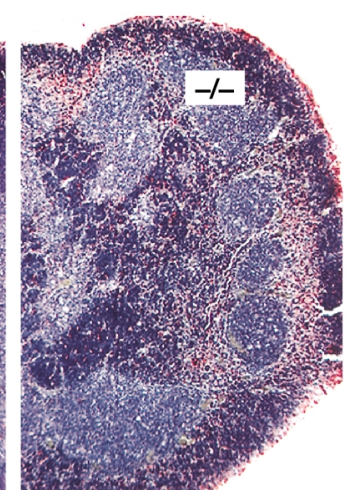

d

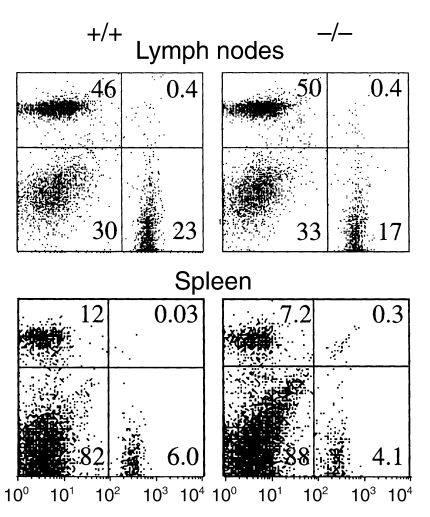

\section{Figure 2}

Increased apoptosis in the thymus but not in the lymph nodes or spleens of $A D A^{-/-}$mice. (a) Top: Side scatter versus forward scatter evaluation of proportion of dead cells in thymus of $A D A^{+/+}$and $A D A^{-/-}$littermates. Numbers indicate percentage of live cells in the lymphoid organ. Bottom: Propidium iodide and Annexin V-aided evaluation of proportion of dead cells in thymus of $A D A^{+/+}$and $A D A^{-/-}$littermates. Numbers indicate the percentage of live cells in the lower left gate estimated using both Annexin $\mathrm{V}$ and PI cell death flow cytometry assay as described in Methods. (b) Cytochemical demonstration of extensive clusters of apoptotic cells in thymus of $A D A^{-/-}$but not of $A D A^{+/+}$littermates. Apoptotic cells are shown in red. The frozen tissue preparations and apoptosis detection were performed as described in Methods. (c) Flow cytometry demonstration of thymocyte distribution in thymi of $A D A^{-/-}$and $A D A^{+/+}$littermates. (d) Flow cytometry demonstration of normal subset distributions in lymph nodes and spleens of $A D A^{-/-}$mice compared with organs of $A D A^{+/+}$littermates. Numbers indicate the percentage of live cells in different subsets estimated as described in Methods. 
but not in secondary immune organs such as the spleen and lymph nodes.

To determine which cells were undergoing apoptosis in $A D A^{-/-}$thymi, the localization of DNA strand breaks in thymus compartments was analyzed by histochemistry in situ. As expected, there was an abundance of apoptosis in $A D A^{-/-}$thymi (Figure $2 \mathrm{~b}$, red). Increased clusters of apoptotic cells were located predominantly in the cortical-medullary boundary (Figure 2b). These findings suggest that immature $T$ cells that accumulate in this area of the thymus are susceptible to the consequences of ADA deficiency.

ADA-deficient mice show abnormal distribution of thymocytes but a relatively normal peripheral $T$ cell distribution. The increased apoptosis at the cortical-medullary boundary of $A D A^{-/-}$thymi prompted us to examine the distribution of lymphocyte populations in this organ. As expected, the majority (70-80\%) of cells recovered from $\mathrm{ADA}^{+/+}$thymi were $\mathrm{CD}^{+} \mathrm{CD}^{+}$double-positive cells (Figure $2 \mathrm{c}$ ). In contrast, the reduction in the doublepositive subset was observed in $A D A^{-/-}$littermates. Although total cell numbers in $A D A^{-/-}$thymi were always dramatically reduced, the decrease in the proportion of DP cell ranged from moderate (Figure 2c, top) to very severe in extreme cases (Figure 2c, bottom). There was a significant increase in the proportion of DN cells ( $24 \%$ vs. $3.2 \%$ in normal thymus), whereas the proportion of DP thymocytes was only moderately decreased (Figure 2c). Analysis of DN thymocytes for the expression of CD44 and CD25 demonstrated no significant differences in $A D A^{+/+}$and $A D A^{-/-}$mice (data not shown). Interestingly, both $\mathrm{CD}^{+}$and $\mathrm{CD}^{+}$singlepositive cells were found in $A D A^{-/-}$thymi, suggesting that a fraction of $A D A^{-/-}$lymphocytes manage to proceed through intrathymic $\mathrm{T}$ cell development (Figure 2c). Consistent with this observation, relatively normal distributions of $\mathrm{CD}^{+}$and $\mathrm{CD}^{+}$lymphocytes were seen in $A D A^{-/-}$lymph nodes and spleens (Figure $2 \mathrm{~d}$ ).

$A D A$ deficiency is accompanied by the presence of $T$ cells with abnormal expression of cell surface markers. Phenotypic changes in peripheral $\mathrm{CD}^{+}$and $\mathrm{CD}^{+} \mathrm{T}$ cells from spleens of $A D A^{-/-}$mice were revealed when ex vivo splenocytes or lymph nodes were analyzed for the expression of different cell surface markers (Figure 3). Surface expression of CD69 and CD44 was increased and CD62L expression was decreased in both $\mathrm{CD}^{+}$and $\mathrm{CD}^{+}$peripheral $A D A^{-/-}$ $\mathrm{T}$ cells. These data suggest that $A D A^{-/-} \mathrm{T}$ cells that manage to escape apoptosis in the thymus are abnormal. Interestingly, expression of CD43 was increased in $A D A^{-/-}$ $\mathrm{CD}^{+} \mathrm{T}$ cells, but was somewhat decreased in $\mathrm{CD}^{+}$cells.

$A D A$ deficiency is accompanied by changes in the distribution of peripheral $B$ cells among marginal, follicular, and newly formed zones in the spleen. To determine the status of $\mathrm{B}$ cells in 3-week-old $A D A^{-/-}$mice, splenic B cells were analyzed using triple staining with mAb's to B220, CD21 and CD23 (27) and to B220, IgM and IgD (data not shown). In these experiments, we found fewer mature B cells among splenocytes mice than in marginal and follicular zones of spleen of $A D A^{-/-}$mice, as was indicated by CD21 versus CD23 analysis (Figure 4b) of $\mathrm{B}^{220^{+}}$splenocytes (Figure 4a).

ADA deficiency is accompanied by TCR activation defects of T cells in vivo. Previously, we demonstrated that adenosine can inhibit $T$ cell activation and expansion in vitro $(6,7,28)$, suggesting that elevated extracellular adenosine levels observed in conditions of ADA deficiency may contribute to the mechanisms of the depletion and function of peripheral lymphocytes by interfering with TCR signaling. To test this hypothesis in vivo, $\mathrm{T}$ cell activation was analyzed in $A D A^{-/-}$mice that exhibited marked increases in circulating adenosine (13). T cell activation in vivo was accomplished by the injection of anti-TCR/CD3 $\mathrm{mAb}$ that is known to activate $\mathrm{T}$ cells both in vitro (29) and in vivo (30). $A D A^{+/+}$ and $A D A^{-/-}$littermates were injected with antiTCR/CD3 mAb, and 16 hours later, T cells were harvested from spleens and $\mathrm{T}$ cell activation was monitored by examining the upregulation of CD25 and
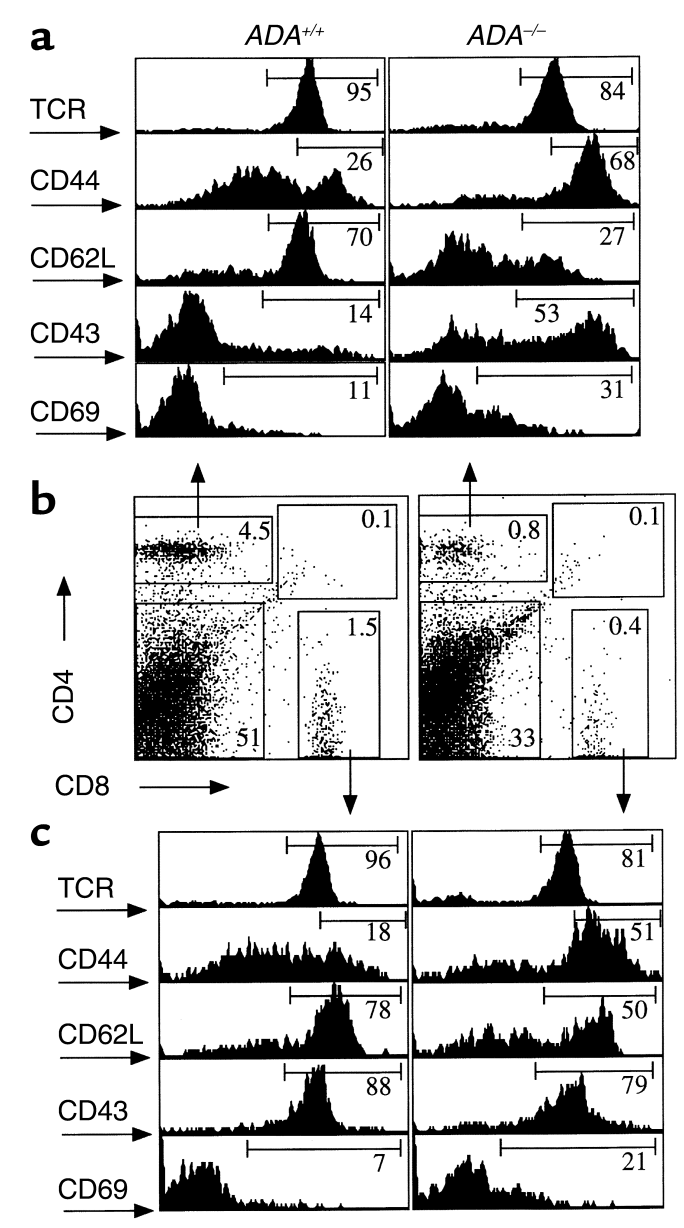

Figure 3

Changes in phenotype of peripheral $\mathrm{CD} 4^{+}$and $\mathrm{CD} 8^{+} \mathrm{T}$ cells from spleens of $A D A^{-/-}$mice. Splenocytes of $A D A^{-/-}$and $A D A^{+/+}$littermates were analyzed by three-color flow cytometry. Expression of different cell surface markers on $\mathrm{CD} 4^{+}$cells $(\mathbf{a})$ and $\mathrm{CD} 8^{+}$cells $(\mathbf{c})$ were analyzed by using CD4/CD8 gates as indicated by arrows in $\mathbf{b}$. Numbers indicate proportions of cell from total cell population (b) or among gated cells ( $\mathbf{a}$ and $\mathbf{c}$ ). 
a

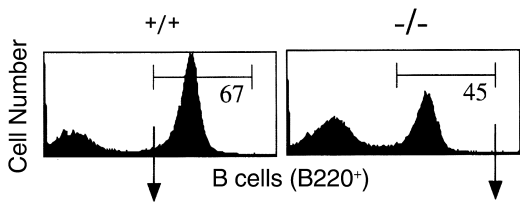

b

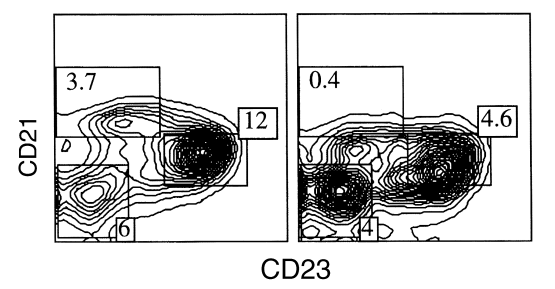

CD23

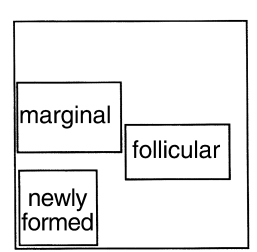

CD69 by flow cytometry. Initial dose-response experiments in control animals suggested that a dose of $5 \mu \mathrm{g}$ of anti-TCR/CD3 mAb was optimal for stimulation of CD25 and CD69 upregulation on T cells (data not shown). This dose was therefore used to assess $\mathrm{T}$ cell activation in $A D A^{-/-}$mice (Figure 5). Pronounced differences in $\mathrm{T}$ cell activation were seen in both $\mathrm{CD} 8^{+}$ (Figure 5a) and $\mathrm{CD}^{+}$(Figure $5 \mathrm{~b}$ ) $\mathrm{T}$ cells from $A D A^{-/-}$ mice. CD25 activation in $\mathrm{CD}^{+} \mathrm{T}$ cells was up to 17 times stronger in $A D A^{+/+}$mice than in $A D A^{-/-}$littermates, and TCR-triggered upregulation of CD69 on $\mathrm{CD}^{+} \mathrm{T}$ cells was approximately two times stronger in $A D A^{+/+}$mice than in $A D A^{-/-}$mice (Figure 5a). Similar differences were seen with $\mathrm{CD} 4^{+} \mathrm{T}$ cells (Figure $5 \mathrm{~b}$ ). These results demonstrated that the activation of both $\mathrm{CD}^{+}$and $\mathrm{CD}^{+} \mathrm{T}$ cells was strongly inhibited in $\mathrm{ADA}^{-/-}$ mice compared with control $A D A^{+/+}$littermates.

Inhibition of TCR-triggered activation of $A D A^{+/+}$ $\mathrm{T}$ cells in vitro requires the addition of ADA inhibitors, suggesting the need to prevent the rapid degradation of adenosine by $\mathrm{ADA}(7)$. To test this interpretation, we incubated ex vivo T cells from $A D A^{+/+}$an $A D A^{-/-}$mice with anti-CD $3 \mathrm{mAb}$ in vitro in the absence of an ADA inhibitor (Figure 6). Results demonstrated that adenosine markedly inhibited TCR-triggered activation in

\section{Figure 4}

Changes in phenotype of peripheral $\mathrm{B}$ cells from spleens of $A D A^{-/-}$mice. Splenocytes of $A D A^{-/-}$and $A D A^{+/+}$littermates were analyzed by three-color flow cytometry as described in Methods and indicated by arrows. (a) Evaluation of $\mathrm{B} 220^{+} \mathrm{B}$ cells in $A D A^{-/-}$and $A D A^{+/+}$littermates. Numbers represent percentage of $B$ cells from total splenocytes. (b) Distribution of B cells among marginal, follicular, and newly formed zones of spleen in $A D A^{-/-}$versus $A D A^{+/+}$mice. Numbers indicate percentage of cells in different zones. Cartoon illustrates location of different $B$ cell subsets according to staining with $\mathrm{mAb}$ to $\mathrm{CD} 21$ and $\mathrm{CD} 23$.
$A D A^{-/-}$but not $A D A^{+/+} \mathrm{T}$ cells. It is shown that control incubations $(0 \mu \mathrm{g}$ of anti-CD3 mAb; Figure 6 , top $)$ had no effect on $\mathrm{CD} 25$ and $\mathrm{CD} 69$ expression in $A D A^{+/+}$or $A D A^{-/-} \mathrm{T}$ cells. In contrast, activation of $\mathrm{T}$ cells with anti-CD3 mAb ( $5 \mu \mathrm{g}$; Figure 6, bottom) resulted in dramatic upregulation of both CD25 and CD69 activation markers. This activation was observed in both $A D A^{+/+}$ and $A D A^{-/-} \mathrm{T}$ cells, although strong activation of $A D A^{-/-} \mathrm{T}$ cells (0.2- $64 \%$ of CD25-expressing cells) was somewhat lower than activation of $A D A^{+/+}$cells (2.6-92\% of CD25-expressing cells).

Importantly, no ADA inhibitors were required to observe the block of TCR-triggered CD25 and CD69 upregulation by adenosine in $A D A^{-/-} \mathrm{T}$ cells, as the proportion of $\mathrm{CD} 25^{+}$cells decreased from $64 \%$ to $2.1 \%$ in the presence of adenosine alone. In contrast, $A D A^{+/+}$ $\mathrm{T}$ cells were affected very little $\left(92 \%\right.$ vs. $88 \%$ of $\mathrm{CD} 25^{+}$ cells in the presence of adenosine). Similar observations of the effects of ADA deficiency and extracellular adenosine were made in evaluations of the CD69 activation marker. These findings demonstrated that activation of $A D A^{-/-} \mathrm{T}$ cells are indeed susceptible to inhibition by elevated adenosine that occurs in ADAdeficient environments, and that effects of ADA inhibitors were indeed due to their ADA-inhibiting a

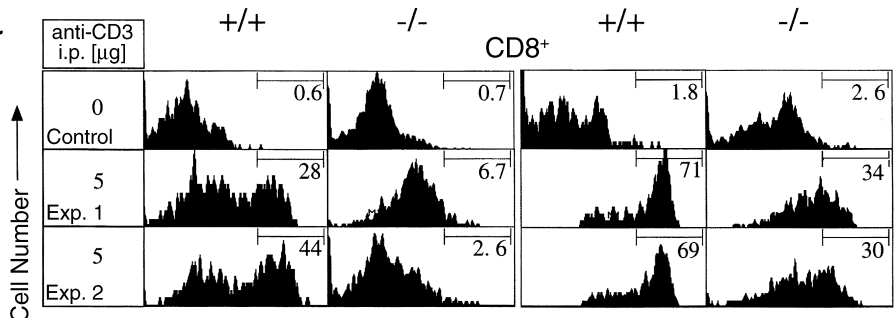

b

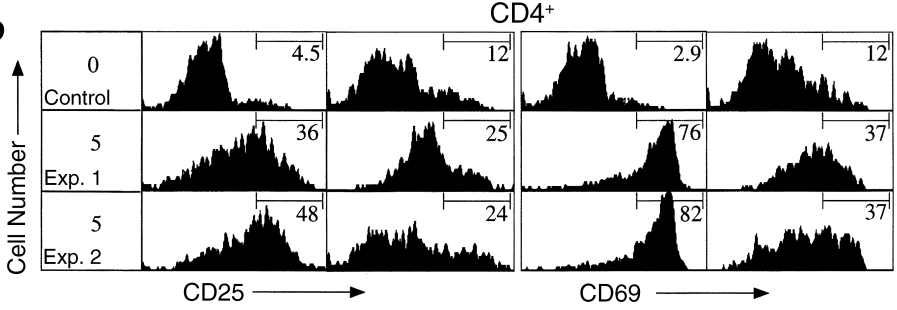

\section{Figure 5}

Inhibited TCR-triggered activation of T cells in an ADAdeficient environment in vivo. $A D A^{+/+}$and $A D A^{-/-}$littermates were injected intraperitoneally (i.p.) with PBS (control) or anti-CD3 mAb; 16 hours later, spleens were harvested and analyzed by flow cytometry for expression of T cell activation markers as described in Methods. Representative results of two (Exp. 1 and Exp. 2) of more than ten similar experiments using more than 20 pairs of littermates of $A D A^{-/-}$and $A D A^{+/+}$ mice are presented. (a) Comparison of TCR-triggered upregulation of CD25 and CD69 surface antigens on CD8 $8^{+} \mathrm{T}$ cells in an $A D A^{-/-}$or $A D A^{+/+}$in vivo environment after injection of $5 \mu \mathrm{g}$ of anti-CD3 mAb. (b) Comparison of TCR-triggered upregulation of CD25 and CD69 surface antigens on CD4 ${ }^{+} T$ cells in an ADAdeficient or ADA-containing in vivo environment after injection of $5 \mu \mathrm{g}$ of anti-CD3 $\mathrm{mAb}$. 

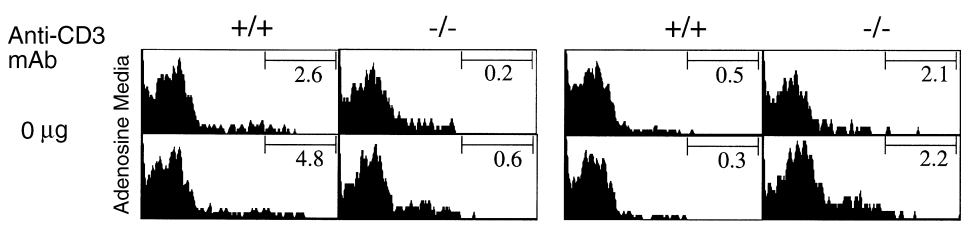

$5 \mu \mathrm{g}$
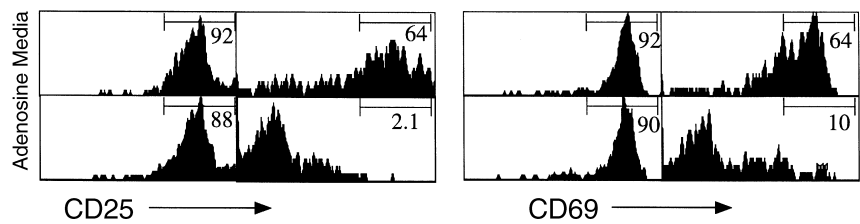

\section{Figure 6}

Effect of adenosine on TCR-triggered upregulation of CD25 and CD69 activation markers in vitro. Ex vivo spleen cells from $A D A^{-/-}$and $A D A^{+/+}$littermates were incubated in 96-well plates with immobilized antiCD3 mAb ( $5 \mu \mathrm{g} / \mathrm{ml} \mathrm{mAb})$ or with serum-free, ADAfree media alone $(0 \mu \mathrm{g} / \mathrm{ml} \mathrm{mAb})$ in the presence or absence of adenosine $(100 \mu \mathrm{M})$; 16 hours later, TCRtriggered upregulation of the $T$ cell activation markers CD69 and CD25 was evaluated by flow cytometry. activities. Another notable observation made in these studies was that a much higher proportion of $A D A^{-/-}$ $\mathrm{T}$ cells were activated in vitro by anti-CD $3 \mathrm{mAb}$ than after anti-CD3 mAb incubation in vivo (cf. Figures 5 and 6 ). For example, about $64 \%$ of $\mathrm{CD}^{+}{ }^{+} \mathrm{T}$ cells from $A D A^{-1-}$ mice were activated by anti-CD3 $\mathrm{mAb}$ in vitro, whereas only $25-36 \%$ of $A D A^{-/-} \mathrm{CD}^{+} \mathrm{T}$ cells were activated by anti-CD3 $\mathrm{mAb}$ in vivo. These findings suggested that the degree of inhibition of TCR-triggered activation of $T$ cells in vivo was likely related to the severity of the metabolic disturbances seen. Collectively, these studies present the first demonstration that TCR-triggered activation of T cells was inhibited in an ADA-deficient environment in vivo.

Adenosine, but not 2'-deoxyadenosine, inhibits TCR-triggered signaling as reflected in the rescue of thymocytes from TCR-induced apoptosis. Adenosine and 2'-deoxyadenosine levels are elevated in ADA-deficient humans and mice $(4,13)$. We proposed recently that TCR-inhibiting properties of extracellular adenosine signaling might contribute to the mechanisms of pathogenesis in ADA SCID (7). The availability of $A D A^{-/-}$mice enabled us to directly test one of the predictions of that model without using ADA inhibitors. Thus, to determine the relative impact of adenosine and 2 -deoxyadenosine on TCR-triggered signaling we selected an assay in which the TCR-inhibiting effects of adenosine would be reflected in protection of thymocytes from cell death. Such a "cell survival" read-out of the experiment on the effects of adenosine was expected to simplify the interpretation of our observations, in that it would allow us

\section{Figure 7}

Extracellular adenosine inhibits TCR-induced signaling in thymocytes. (a) Adenosine alone inhibits TCR-triggered apoptosis in $A D A^{-/-}$but not in normal $A D A^{+/+}$thymocytes. (b) Demonstration of opposite effects of adenosine and 2 -deoxyadenosine on spontaneous and TCR-triggered apoptosis in $A D A^{-/-}$thymocytes. Thymocytes from wild-type or $A D A^{-/-}$mice were incubated for 16 hours in 96-well plates with apoptosis-inducing immobilized anti-CD3 mAb in the presence or absence of added adenosine $(100 \mu \mathrm{M})$ or $2^{\prime}$ deoxyadenosine $(100 \mu \mathrm{M})$. The effect of adenosine and $2^{\prime}$ deoxyadenosine on thymocyte survival (proportion of live cells) was evaluated using Annexin $\mathrm{V}$ assay as described in the Methods. to discount possible toxic effects of intracellular adenosine and of its metabolites. Figure 7 demonstrates the effects of exogenous adenosine or 2'-deoxyadenosine on $\mathrm{T}$ cells during their incubation with anti-CD3 $\mathrm{mAb}$ in vitro. Incubation of $A D A^{+/+}$thymocytes in vitro resulted in some "spontaneous" cell death (Figure 7a), and the addition of anti-CD3 mAb increased the amount of cell death seen. Addition of adenosine did not affect the outcome of CD3-induced apoptosis in $A D A^{+/+}$thymocytes, most likely because the added adenosine was degraded by ADA. In contrast, the same concentration of exogenously added adenosine was very efficient in blocking TCR-signaling, and protected $A D A^{-/-}$thymocytes from both spontaneous and TCR-triggered apoptosis (Figure 7a, -/- graph).

In contrast to adenosine, we found that $2^{\prime}$ deoxyadenosine (Figure $7 \mathrm{~b}$ ) not only did not inhibit TCR-induced apoptosis of thymocytes, but it strongly

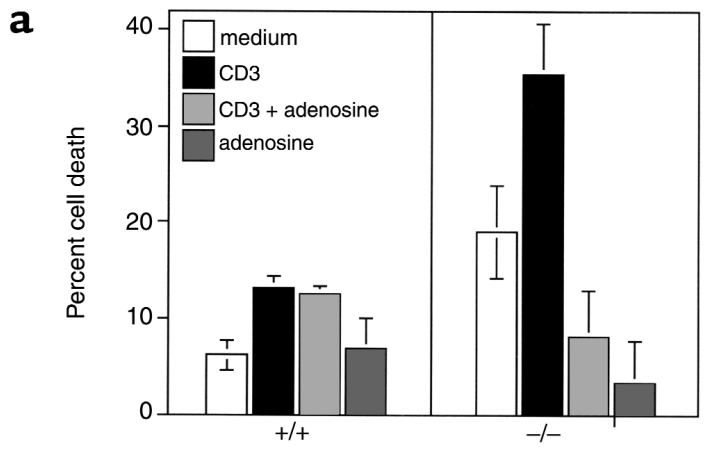

b

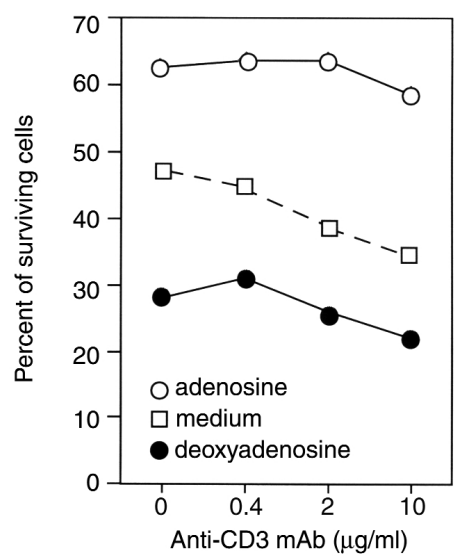




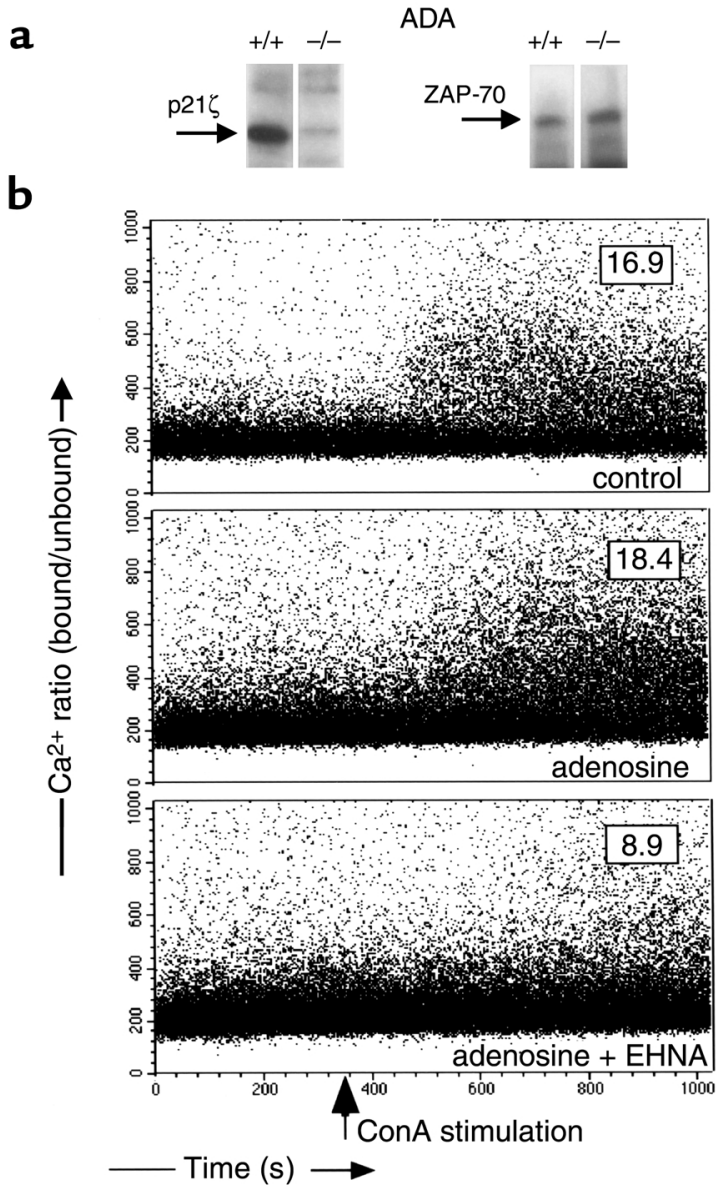

Figure 8

ADA deficiency is accompanied with defects in TCR signaling pathways in vivo and in vitro. (a) Partial phosphorylation of CD3 $\zeta$ chain is notably reduced in nonactivated ex vivo thymocytes from $A D A^{-/-}$ mice, whereas ZAP-70 levels are shown to be the same in parallel samples of immunoprecipitates from ex vivo thymocytes harvested from $A D A^{-/-}$and $A D A^{+/+}$littermates. (b) Intracellular $\mathrm{Ca}^{2+}$ mobilization upon ConA stimulation is inhibited by adenosine in normal thymocytes in the presence of the ADA inhibitor EHNA. Thymocytes from $A D A^{+/+}$or $A D A^{-/-}$mice were used for isolation of ZAP-70 immunocomplexes followed by immunoblotting analysis of TCR $\zeta$ chain phosphorylation with an anti phosphotyrosine $\mathrm{mAb}$ as described in Methods. Anti ZAP-70 mAb's were used in control immunoblotting. For measurements of calcium flux $A D A^{+/+}$thymocytes were preloaded with indo- 1 and analyzed on a FACSVantage flow cytometer as described in Methods. The percentages of cells that increase intracellular calcium after stimulation with Concanavalin $\mathrm{A}$ are shown on the graphs. Adenosine $(100 \mu \mathrm{M})$ alone or in combination with EHNA $(10 \mu \mathrm{M})$ was added a minute before the ConA stimulation. Arrow indicates time of injection of T cell-activating stimuli.

exacerbated both spontaneous and TCR-triggered apoptosis. These data confirmed the well-documented observations of $2^{\prime}$-deoxyadenosine lymphotoxicity and suggested that $2^{\prime}$-deoxyadenosine was not involved in the inhibition of TCR-triggered events in surviving $\mathrm{T}$ cells in $A D A^{-/-}$mice or in experiments in vitro.

ADA deficiency is accompanied by defects in TCR signaling pathways in vivo and in vitro. Proper TCR signaling plays a critical role in survival and development of thymo- cytes in vivo. It has been extensively shown that TCR molecules trigger a complex series of early and late biochemical events including tyrosine phosphorylation and $\mathrm{Ca}^{2+}$ mobilization $(20,21,24,31$,). It was therefore important to determine whether the inhibitory function of adenosine in $A D A^{-/-}$thymocytes was the result of inhibition in TCR-triggered biochemical events in vivo. A number of studies have demonstrated the importance of the TCR $\zeta$ chain phosphorylation for initiating of TCR cell signaling. In primary thymic and lymph node T cells, the TCR $\zeta$ chain appears to be preassociated with ZAP-70, which plays a central role in the $T$ cell signal transduction $(32,33)$. Using approaches developed in studies of phosphotyrosine phosphorylation of TCR-associated signaling CD3 chains (24, 31 , we compared the level of $\zeta$ p21 tyrosine phosphorylation in ex vivo thymocytes from $A D A^{-/-}$and $A D A^{+/+}$ control littermates. Partial phosphorylation of $\mathrm{CD} 3 \zeta$ chain was notably reduced in nonactivated ex vivo thymocytes from $A D A^{-/-}$mice (Figure $8 \mathrm{a}$ ), suggesting that an ADA-deficient environment in vivo is not conducive to normal levels of TCR signaling. An internal biochemical control was provided by blotting of the same samples with anti-ZAP-70 mAb's to show similar amount of ZAP-70 in immunoprecipitated samples from both $A D A^{-/-}$and $A D A^{+/+}$littermates (Figure 8a).

Observations of inhibited TCR-CD3 chain tyrosine phosphorylation most likely reflects inhibited TCR signaling in the in vivo thymic environment of $A D A^{-/-}$mice and predicts that other early events of TCR signaling (e.g., intracellular $\mathrm{Ca}^{2+}$ accumulation) may be inhibited by adenosine in conditions of ADA deficiency. This was examined by in vitro studies of intracellular $\mathrm{Ca}^{2+}$ accumulation in activated $A D A^{+/+}$thymocytes in the presence of the ADA inhibitor EHNA (Figure 8b). The addition of adenosine or EHNA alone did not have an inhibitory effect on $\mathrm{Ca}^{2+}$ levels in this short term $\mathrm{Ca}^{2+}$ measurement assay (Figure 8b and data not shown). However, the addition of adenosine in the presence of EHNA inhibited $\mathrm{Ca}^{2+}$ accumulation (Figure 8b, bottom). Adenosine alone was able to inhibit $\mathrm{Ca}^{2+}$ mobilization in a similar experiment with $A D A^{-/-}$thymocytes, thereby confirming that effects of EHNA were due to its interactions with ADA (data not shown). Thus, early events of TCR-mediated signaling in thymocytes are inhibited in an ADA-deficient environment.

\section{Discussion}

ADA deficiency in humans results in an immunodeficiency characterized by a severe reduction in $\mathrm{T}, \mathrm{B}$, and NK cells (3). The generation of $A D A^{-/-}$mice (13) has allowed the examination of molecular mechanisms that underlie the immunodeficiency seen in ADA-deficient humans. In this study, we demonstrated that there was a severe lymphopenia in $A D A^{-/-}$mice at 3 weeks of age. Furthermore, there was an abundance of apoptosis occurring in thymi of $A D A^{-/-}$mice, but not in the spleen and lymph nodes of these animals. These findings provided evidence that the metabolic disturbances associat- 
ed with ADA deficiency induced apoptosis in developing thymocytes in vivo. Peripheral $\mathrm{T}$ and $\mathrm{B}$ cells were abnormal in $A D A^{-/-}$mice as reflected in the expression of cell surface markers and localization in different zones of lymphoid organs. In addition, mature $\mathrm{T}$ cells recovered from spleens of $A D A^{-/-}$mice were defective with regard to their ability to functionally signal through the TCR. Finally, ex vivo experiments on $A D A^{-/-} \mathrm{T}$ cells demonstrated that elevated adenosine was responsible for inhibited and abnormal TCR signaling.

The immunodeficiency seen in ADA-deficient patients can be severe $(3,4)$, suggesting mature lymphocytes and/or their precursors are sensitive to the metabolic disturbances associated with ADA deficiency. Correspondingly, studies have demonstrated that apoptosis can be induced in lymphocytes after direct exposure to adenosine or $2^{\prime}$-deoxyadenosine in vitro $(25,26)$. Our ability to analyze apoptosis in the immune system of $A D A^{-/-}$mice has provided the first in vivo evidence that apoptosis is abundant in $A D A^{-/-}$ thymi. Most of the apoptosis seen in $A D A^{-/-}$thymi was localized to the cortical-medullary boundary, a location where $\mathrm{CD} 4^{+} \mathrm{CD}^{+}$double-positive thymocytes should accumulate. Consistent with these findings, analysis of thymocyte populations in these and previous studies (13) show a reduction of in the number of $\mathrm{CD} 4^{+} \mathrm{CD}^{+}$ thymocytes in $A D A^{-/-}$mice. Similar findings have been described in mice treated with the ADA inhibitor deoxycoformycin (26). Interestingly, increased apoptosis was not noticed in histological studies and lymphocytes collected from $A D A^{-/-}$spleens or lymph nodes (data not shown), suggesting that mature lymphocytes are less sensitive to the cytotoxic properties of adenosine or 2'-deoxyadenosine. Indeed, in vitro observations suggest that mature $\mathrm{CD}^{+} \mathrm{T}$ cells do not undergo apoptosis in response to $2^{\prime}$-deoxyadenosine exposure (26). These data suggest that $\mathrm{CD} 4^{+} \mathrm{CD} 8^{+}$double-positive thymocytes, or their immediate precursors, are sensitive to the metabolic disturbances seen in ADA deficiency, and the depletion of this population is likely to account for the reduction of mature $\mathrm{T}$ cells found in the periphery of ADA-deficient mice (13) and humans $(3,4)$. Individual variations in ADA levels during early $A D A^{-/-}$mice development may account for the severity of apoptotic and phenotypic changes in thymus. Drastic reduction with almost disappearance of double-positive thymocytes in some litters (Figure 2c) after 3 weeks of postnatal development could be the result of low level expression of ADA minigene in the progeny from particular breeders, and the exact mechanism of it is under study (S.G. Apasov, work in progress).

Both adenosine and 2'-deoxyadenosine exhibit potent biologic activities that may impact $T$ cell development and function. Adenosine serves as a extracellular signaling molecule by engaging $G$ protein-coupled receptors on the surface of target cells (5), whereas $2^{\prime}$-deoxyadenosine is cytotoxic to cells through mechanisms that include the inhibition of cellular transmethylation reactions (34), the disrup- tion of cellular growth $(10,11)$ or differentiation $(35)$ and the activation of apoptosis (12). The large amount of apoptosis detected in the thymi of $A D A^{-/-}$mice, recent studies in fetal thymic organ cultures (36) and the observation of 2 -deoxyadenosine accumulation in the thymus of $A D A^{-/-}$mice $(13,15)$ support the view that ADA substrates generated from apoptotic thymocytes contribute to the cytotoxicity and phenotypic outcomes of ADA deficiency.

Additionally, our findings suggest that the metabolic disturbances associated with ADA deficiency impact both intrathymic $\mathrm{T}$ cell development and mature $\mathrm{T}$ cell functions. We had shown previously that $\mathrm{T}$ cells treated with a combination of ADA inhibitors and adenosine in vitro exhibit defects in TCR induced activation $(6,7,8,28)$. In this study, we extended these observations to show similar effect on $A D A^{-/-} \mathrm{T}$ cells, which challenged with anti-CD3 $\mathrm{mAb}$ in vivo. Both $\mathrm{CD} 4^{+}$and $\mathrm{CD}^{+}$cells in $\mathrm{ADA}^{-/-}$mice had a decreased capacity to upregulate $\mathrm{CD} 25$ and $\mathrm{CD} 69$ expression in response to anti-CD3 mAb in vivo. In important control experiments, these cells were activated by anti-CD3 $\mathrm{mAb}$ in vitro similarly to wild type, whereas added adenosine was inhibitory to the activation of $A D A^{-/-}$but not $A D A^{+/+} \mathrm{T}$ cells. These findings suggest that the increased levels of adenosine found in peripheral lymphoid organs such as the spleen (13) can affect normal $T$ cell signaling. Disruptions in normal $T$ cell signaling could in turn affect the function of existing peripheral $\mathrm{T}$ cells in $A D A^{-/-}$mice, by preventing $\mathrm{T}$ cell activation during an immune response.

These observations also suggest that the T cell depletion in ADA SCID may be at least partially due to blocks in TCR-driven thymocyte maturation by adenosine, as well as to direct apoptotic effects of intracellular adenosine, $2^{\prime}$-deoxyadenosine, and dATP. Thus, we propose that there may be at least two alternative or simultaneously operating mechanisms of $T$ cell depletion: (a) intracellular lymphotoxicity of intracellularly accumulated adenosine, 2 '-deoxyadenosine, and dATP $(4,9,10)$ and (b) inhibition of TCR signaling and, hence, the inhibition/block of TCR-driven processes of $T$ cell selection $(7,8)$. Both of these mechanisms have potential to cause $\mathrm{T}$ cell depletion in our studies in $A D A^{-/-}$mice and in patients with ADA SCID.

The challenging task for testing the signaling model of ADA SCID was in demonstrating that the inhibition of TCR signaling by extracellular adenosine was not due to translocation of exogenously added adenosine into the cytoplasm followed by intracellular lymphotoxicity of adenosine catabolites. One way to exclude considerations of lymphotoxicity of exogenously added adenosine was to use an assay system in which adenosine was rescuing $T$ cells from death by virtue of acting as a TCR-antagonizing ligand. Accordingly, we used an assay in which incubation of TCR-transgenic thymocytes with antigenic peptides and antigen-presenting cells resulted in their activation followed by their apoptosis (7). The demonstrated rescue of thymocytes from 
TCR-induced death (7) by adenosine was evidence of the ability of extracellular adenosine to antagonize the TCR-signaling and TCR-driven processes in conditions of ADA deficiency without being toxic in vitro. It was, however, still possible, that there was not enough extracellular adenosine to block $\mathrm{T}$ cell activation in an ADAdeficient environment in vivo. This issue could not be addressed without $A D A^{-/-}$mice, and in this report, evidence in support of this was provided by showing that there was a strong inhibition of a $\mathrm{T}$ cell response in $A D A^{-/-}$mice after injection with activating anti-TCR$\mathrm{CD} 3 \mathrm{mAb}$ (Figure 5). Taken together, these findings suggest that the metabolic disturbances seen in ADAdeficient mice are capable of affecting various signaling pathways and intracellular processes that regulate thymocyte survival and function.

In conclusion, we have demonstrated that ADA deficiency in mice severely effects intrathymic $T$ cell development and TCR-mediated signaling in the periphery. Our results show that ADA deficiency is associated with impaired TCR signaling and enhanced thymocyte apoptosis. This observations suggest a model that inhibition of TCR signaling in developing thymocytes may block positive selection resulting in the increase of cell death among immature thymocytes. This, in turn, will increase the accumulation of extracellular adenosine and 2 '-deoxyadenosine facilitating apoptotic process in the thymus. Although the exact mechanisms by which the ADA substrates adenosine and 2 '-deoxyadenosine promote immunodeficiency as a whole are still not clear, the data presented in this in vivo model have provided insight that may not have been previously appreciated. The observation that $2^{\prime}$-deoxyadenosine accumulation is greatest in the thymus (15), and the visualization of massive apoptosis in this organ, provide strong correlative evidence that this metabolite mediates apoptosis in this organ. The widespread accumulation of adenosine, together with the observation that adenosine inhibits TCR-triggered activation events in immature and mature T cells, suggest that systemic accumulations of adenosine may prevent the TCR signaling-dependent thymocyte differentiation and normal function of peripheral $\mathrm{T}$ cells. This model lends itself well to additional genetic manipulations that will help to determine the specific mechanisms involved in these processes. In addition, the ability to perform ADA enzyme therapy and gene therapy in this model (37) will provide a means to further assess the lymphoid specificity of this disease and the efficacy of these novel therapies.

\section{Acknowledgments}

The authors thank Andrea Barnes, Michelle Kress, and Charlene Shaw (National Institute of Allergy and Infectious Diseases, NIH) for help with breeding and screening of mice, Brenda Marshall for editing, and Shirley Starnes for preparation of the manuscript. Special thanks to I. Stefanova for help with immunoblotting. This work was supported in part by NIH grants
(DK46207 and DK54443 to R.E. Kellems; and AI43572 and HL61888 to M.R. Blackburn).

1. Giblett, E.R., Anderson, J.E., Cohen, F., Pollara, B., and Meuwissen, H.J. 1972. Adenosine-deaminase deficiency in two patients with severely impaired cellular immunity. Lancet. 2:1067-1069.

2. Giblett, E.R., Ammann, A.J., Wara, D.W., Sandman, R., and Diamond, L.K. 1975. Nucleoside-phosphorylase deficiency in a child with severely defective T-cell immunity and normal B-cell immunity. Lancet. 1:1010-1013.

3. Buckley, R.H., et al. 1997. Human severe combined immunodeficiency: genetic, phenotypic, and functional diversity in one hundred eight infants. J. Pediatr. 130:378-387.

4. Hershfield, M.S., and Mitchell, B.S. 1995. Immunodeficiency diseases caused by adenosine deaminase deficiency and purine nucleoside phosphorylase deficiency. In The molecular and metabolic bases of inherited disease. C.R. Scriver, A.L. Beaudet, W.S. Sly, and D. Valle, editors. McGraw-Hill. New York, New York, USA. 1725-1768.

5. Olah, M.E., and Stiles, G.L. 1995. Adenosine receptor subtypes: characterization and therapeutic regulation. Annu. Rev. Pharmacol. Toxicol. 35:581-606.

6. Koshiba, M., Kojima, H., Huang, S., Apasov, S., and Sitkovsky, M.V. 1997. Memory of extracellular adenosine $A_{2 A}$ purinergic receptor-mediated signaling in murine T cells. J. Biol. Chem. 272:25881-25889.

7. Apasov, S.G., and Sitkovsky, M.V. 1999. The extracellular versus intracellular mechanisms of inhibition of TCR- triggered activation in thymocytes by adenosine under conditions of inhibited adenosine deaminase. Int. Immunol. 11:179-189.

8. Apasov, S.G., Chen, J.F., Smith, P.T., and Sitkovsky, M.V. 2000. A $2 \mathrm{~A}$ receptor dependent and $\mathrm{A}_{2 \mathrm{~A}}$ receptor independent effects of extracellular adenosine on murine thymocytes in conditions of adenosine deaminase deficiency. Blood. 95:3859-3867.

9. Hershfield, M.S., Kredich, N.M., Ownby, D.R., Ownby, H., and Buckley, R. 1979. In vivo inactivation of erythrocyte S-adenosylhomocysteine hydrolase by 2'-deoxyadenosine in adenosine deaminase-deficient patients. J. Clin. Invest. 63:807-811.

10. Ullman, B., Gudas, L.J., Cohen, A., and Martin, D.W., Jr. 1978. Deoxyadenosine metabolism and cytotoxicity in cultured mouse $\mathrm{T}$ lymphoma cells: a model for immunodeficiency disease. Cell. 14:365-375.

11. Carson, D.A., et al. 1985. Metabolic basis for immune dysfunction in adenosine deaminase deficiency. Ann. NY Acad. Sci. 451:34-41.

12. Liu, X., Kim, C.N., Yang, J., Jemmerson, R., and Wang, X. 1996. Induction of apoptotic program in cell-free extracts: requirement for dATP and cytochrome c. Cell. 86:147-157.

13. Blackburn, M.R., Datta, S.K., and Kellems, R.E. 1998. Adenosine deaminase-deficient mice generated using a two-stage genetic engineering strategy exhibit a combined immunodeficiency. J. Biol. Chem. 273:5093-5100.

14. Blackburn, M.R., Wakamiya, M., Caskey, C.T., and Kellems, R.E. 1995. Tissue-specific rescue suggests that placental adenosine deaminase is important for fetal development in mice. J. Biol. Chem. 270:23891-23894.

15. Blackburn, M.R., Datta, S.K., Wakamiya, M., Vartabedian, B.S., and Kellems, R.E. 1996. Metabolic and immunologic consequences of limited adenosine deaminase expression in mice. J. Biol. Chem. 271:15203-15210.

16. Knudsen, T.B., Blackburn, M.R., Chinsky, J.M., Airhart, M.J., and Kellems, R.E. 1991. Ontogeny of adenosine deaminase in the mouse decidua and placenta: immunolocalization and embryo transfer studies. Biol. Reprod. 44:171-184.

17. Apasov, S.G., Koshiba, M., Chused, T.M., and Sitkovsky, M.V. 1997. Effects of extracellular ATP and adenosine on different thymocyte subsets: possible role of ATP-gated channels and G protein-coupled purinergic receptor. J. Immunol. 158:5095-5105.

18. Darzynkiewicz, Z., et al. 1992. Features of apoptotic cells measured by flow cytometry. Cytometry. 13:795-808.

19. Martin, S.J., et al. 1995. Early redistribution of plasma membrane phosphatidylserine is a general feature of apoptosis regardless of the initiating stimulus: inhibition by overexpression of Bcl-2 and Abl. J. Exp. Med. 182:1545-1556.

20. Rabinovitch, P.S., June, C.H., Grossmann, A., and Ledbetter, J.A. 1986. Heterogeneity among $\mathrm{T}$ cells in intracellular free calcium responses after mitogen stimulation with PHA or anti-CD3. Simultaneous use of indo-1 and immunofluorescence with flow cytometry. J. Immunol. 137:952-961.

21. Rabin, R.L., et al. 1999. Chemokine receptor responses on T cells are achieved through regulation of both receptor expression and signaling. J. Immunol. 162:3840-3850.

22. Modak, S.P., and Bollum, F.J. 1972. Detection and measurement of single-strand breaks in nuclear DNA in fixed lens sections. Exp. Cell Res. 75:307-313. 
23. Ben-Sasson, S.A., Sherman, Y., and Gavrieli, Y. 1995. Identification of dying cells: in situ staining. Methods Cell Biol. 46:29-39.

24. Hemmer, B., Stefanova, I., Vergelli, M., Germain, R.N., and Martin, R. 1998. Relationships among TCR ligand potency, thresholds for effector function elicitation, and the quality of early signaling events in human T cells. J. Immunol. 160:5807-5814.

25. Kizaki, H., Suzuki, K., Tadakuma, T., and Ishimura, Y. 1990. Adenosine receptor-mediated accumulation of cyclic AMP-induced T-lymphocyte death through internucleosomal DNA cleavage. J. Biol. Chem. 265:5280-5284.

26. Benveniste, P., and Cohen, A. 1995. p53 expression is required for thymocyte apoptosis induced by adenosine deaminase deficiency. Proc Natl. Acad. Sci. USA. 92:8373-8377.

27. Oliver, A.M., Martin, F., Gartland, G.L., Carter, R.H., and Kearney, J.F. 1997. Marginal zone B cells exhibit unique activation, proliferative and immunoglobulin secretory responses. Eur. J. Immunol. 27:2366-2374.

28. Huang, S., Apasov, S., Koshiba, M., and Sitkovsky, M. 1997. Role of A2a extracellular adenosine receptor-mediated signaling in adenosine-mediated inhibition of T-cell activation and expansion. Blood. 90:1600-1610.

29. Ferran, C., Bach, J.F., and Chatenoud, L. 1993. In vivo T cell activation properties of anti-T cell monoclonal antibodies. Exp. Nephrol. 1:83-89.

30. Bluestone, J.A., Bruce, D.S., and Newell, K.A. 1992. Activation of T cells in vivo using anti-CD3 and staphylococcal enterotoxins. Int. J. Cancer Suppl. 7:39-41.

31. Burkhardt, A.L., et al. 1994. Temporal regulation of non-transmembrane protein tyrosine kinase enzyme activity following $\mathrm{T}$ cell antigen receptor engagement. J. Biol. Chem. 269:23642-23647.

32. Sebzda, E., et al. 1999. Selection of the T cell repertoire. Annu. Rev. Immunol. 17:829-874.

33. Bolen, J.B., and Brugge, J.S. 1997. Leukocyte protein tyrosine kinases: potential targets for drug discovery. Annu. Rev. Immunol. 15:371-404.

34. Hershfield, M.S., and Kredich, N.M. 1980. S-Adenosylhomocysteine metabolism in adenosine deaminase deficient cells. Adv. Exp. Med. Biol. 122A:421-425.

35. Benveniste, P., Zhu, W., and Cohen, A. 1995. Interference with thymocyte differentiation by an inhibitor of S- adenosylhomocysteine hydrolase. J. Immunol. 155:536-544.

36. Thompson, L.F., et al. 2000. Metabolites from apoptotic thymocytes inhibit thymopoiesis in adenosine deaminase-deficient fetal thymic organ cultures. J. Clin. Invest. 106:1149-1157.

37. Blackburn, M.R., et al. 2000. The use of enzyme therapy to regulate the metabolic and phenotypic consequences of adenosine deaminase deficiency in mice: differential impact on pulmonary and immunologic abnormalities. J. Biol. Chem. 275:32114-32121. 